\title{
Guided by Evolution: Biology-Oriented Synthesis of Bioactive Compound Classes
}

\author{
George Karageorgis ${ }^{a, b}$ \\ Herbert Waldmann*a (iD \\ a Max-Planck-Institute of Molecular Physiology, \\ Department of Chemical Biology, Otto-Hahn-str. \\ 11, 44227 Dortmund, Germany \\ herbert.waldmann@mpi-dortmund.mpg.de \\ ${ }^{b}$ Current address: School of Chemistry, University of \\ Leeds, Woodhouse Lane, Leeds, LS2 9JT, UK \\ Published as part of the 50 Years SYNTHESIS - \\ Golden Anniversary Issue
}
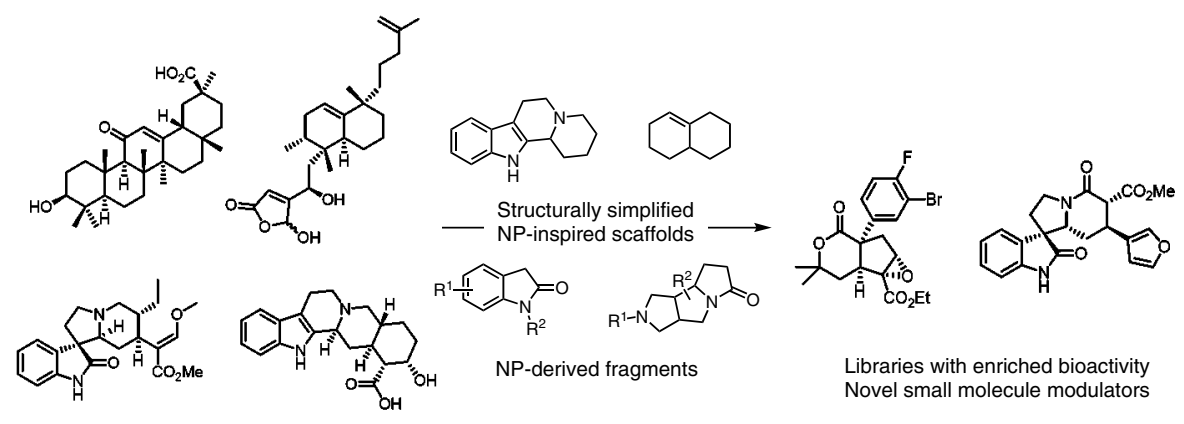

Libraries with enriched bioactivity Novel small molecule modulators

\author{
Received: 29.08.2018 \\ Accepted: 03.09.2018 \\ Published online: 11.10 .2018 \\ DOI: 10.1055/s-0037-1610368; Art ID: ss-2018-z0577-sr \\ License terms: CCO
}

\begin{abstract}
Biology-oriented-synthesis (BIOS), is a chemocentric approach to identifying structurally novel molecules as tools for chemical biology and medicinal chemistry research. The vast chemical space cannot be exhaustively covered by synthetic chemistry. Thus, methods which reveal biologically relevant portions of chemical space are of high value. Guided by structural conservation in the evolution of both proteins and natural products, BIOS classifies bioactive compound classes in a hierarchical manner based on molecular architecture and bioactivity. Biologically relevant scaffolds inspire and guide the synthesis of BIOS libraries, which calls for the development of suitable synthetic methodologies. These compound collections have enriched biological relevance, leading to the discovery of bioactive small molecules. These potent and selective modulators allow the study of complex biological pathways and may serve as starting points for drug discovery programs. Thus, BIOS can also be regarded as a hypothesis-generating tool, guiding the design and preparation of novel, bioactive molecular scaffolds. This review elaborates the principles of BIOS and highlights selected examples of their application, which have in turn created future opportunities for the expansion of BIOS and its combination with fragment-based compound discovery for the identification of biologically relevant small molecules with unprecedented molecular scaffolds. 1 Introduction

2 Structural Classification of Natural Products

3 Implications and Opportunities for Biology-Oriented Synthesis

4 Applications of Biology-Oriented Synthesis

4.1 Chemical Structure and Bioactivity Guided Approaches

4.2 Natural-Product-Derived Fragment-Based Approaches

5 Conclusions and Outlook
\end{abstract}

Key words natural products, bioorganic chemistry, stereoselective synthesis, high-throughput screening, combinatorial chemistry

\section{Introduction}

Small molecules are excellent tools for chemical biology research and the analysis of complex biological mechanisms. Their effect is acute, yet not permanent and can be tuned, e.g., by dosage, to work reversibly and rapidly, ${ }^{1}$ in contrast to most genetic approaches. Thus, the development of selective small molecule as probes for protein targets is of high value for studying and understanding biolog-
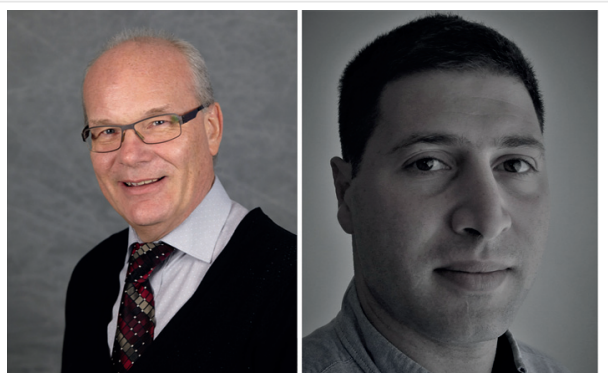

Herbert Waldmann studied chemistry at the University of Mainz, where he received his $\mathrm{PhD}$ in organic chemistry in 1985 under the guidance of Prof. Horst Kunz. After a postdoctoral appointment with Prof. G. Whitesides at Harvard University, he completed his habilitation at the University of Mainz in 1991. In 1999 he was appointed Director at the Max Planck Institute of Molecular Physiology, Dortmund, and Full Professor of Biochemistry at the University of Dortmund. His research focuses on the syntheses of signal-transduction modulators and natural-product-inspired compound libraries and their biological evaluation. Dr George Karageorgis was born in Nicosia, Cyprus, and graduated from the Aristotle University of Thessaloniki, Greece, with a B.Sc. Chemistry degree in 2010 and obtained a M.Sc. in Chemical Biology from the University of Leeds in 2011. He joined Prof. A. Nelson's group in Leeds as a PhD student where he worked on the development of activity-directed synthesis. He was awarded an Alexander von Humboldt Fellowship to join Prof. H. Waldmann's group at the Max-Planck Institute in Dortmund, working on the design and syntheses of biologically relevant small molecules with novel molecular scaffolds. 
ical phenomena. It is estimated that drug-like chemical space may accommodate ca. $10^{60}$ small molecules, ${ }^{2}$ rendering its investigation by means of synthesis alone an unfeasible task.

On the other end of the scale, nature has circumvented this issue by conserving the structural and chemical properties of protein-binding sites during their selection and evolution. There are more than $10^{390}$ combinations possible for a protein of an average size of 300 amino acids, synthesized from 20 different amino acids. ${ }^{2}$ However, an estimated 25,000 proteins may be encoded even in the complicated human genome, containing subdomains that are conserved within protein classes. This restriction may be attributed to thermodynamic stability rather than function, which imposes physical and chemical evolutionary pressure on protein formation., ${ }^{3,4}$ The arrangement of secondary structures ( $\alpha$ helices and $\beta$ sheets) determines the three-dimensional structure of proteins, which leads to the formation of characteristic fold classes of the individual protein domains. These folds determine the size and shape of binding sites for ligands or substrates, whilst the chemical structure of amino acid residue side chains determines the kind of ligand (e.g., polar, nonpolar) that can be bound. Additionally, these ligand-accommodating sites within single domain proteins can be represented by about 1300 binding pockets. ${ }^{3}$ This fact suggests that a given fold type may be formed by different amino acid sequences and thus even protein domains with low primary sequence homology can have very similar folds. ${ }^{5}$

In direct analogy, natural products (NPs) have a limited number of core scaffolds, which are distinguished by different groups decorating the central scaffold. NPs are smallmolecule secondary metabolites and have been a major source of inspiration for chemists and chemical biologists. ${ }^{6}$ NPs are intrinsically different from compounds resulting from conventional combinatorial synthesis, due to their increased three-dimensional character, which also correlates with higher success rates in drug discovery projects. ${ }^{7}$ NPs have evolved to interact with more than one protein, as they are typically formed through sequential binding of biosynthetic intermediates to different enzymes and also display different biological effects, either within the producing organism or across different species. ${ }^{8}$ The diverse substitution patterns in NPs of related molecular scaffolds result in different bioactivity profiles, suggesting that NPs define evolutionary selected structures, which can interact with multiple proteins, whilst selectivity is finely tuned by the functional groups of each individual molecule. NPs define biologically relevant portions of vast chemical space.

These principles can be applied in the design and preparation of novel small molecules which modulate the function and activity of proteins. NP molecular scaffolds can be analyzed to identify the relevant features for bioactivity and subsequently applied to the synthesis of NP-inspired compound libraries with simplified, more synthetically attain- able structures. Systematic and structure-based approaches, which can serve as the basis and guide the development of small molecules with novel bioactivities, are of high value in chemical biology and medicinal chemistry research. Biology-oriented synthesis (BIOS) is based on the structural analyses of NPs and their respective protein targets and abides to the evolutionary conservatism and functionalgroup diversity principles encountered in nature. Developing collections of NP-inspired compounds with diverse substitution patterns around a common core scaffold may result in matching the diversity of amino acid side chains occurring within otherwise similar protein subfolds. Thus, BIOS is a chemocentric approach taking advantage of prevalidated, biologically relevant starting points offered by NP structure. The resulting compound collections are enriched in bioactivity and can be used for the development of molecular tools for the study of protein function and to decipher complex biological phenomena.

\section{Structural Classification of Natural Prod- ucts}

To chart the chemical space covered by NPs, a chemoinformatic tool termed 'structural classification of natural products' (SCONP) was developed. ${ }^{9}$ By using SCONP, the highly complex NP structures can be reduced, by classifying and arranging the core scaffolds in a hierarchical manner, resulting in the generation of a branch for each scaffold. In each branch, each parent scaffold is a substructure of its offspring and branches merge together to generate a scaffold tree (Figure 1). Initially, the chemical space covered by natural products contained gaps where certain substructures had not been discovered yet or had not emerged through nature's evolutionary process. Virtual scaffolds were thus generated in silico, derived from the iterative deconstruction process itself. These scaffolds have not yet been reported and they provide excellent starting points for the syntheses of NP-inspired compounds.

Moving along the branches of the scaffold tree, starting from the outer, more complex structures to the inner, structurally more simplified scaffolds is a process termed 'brachiation'. This linear movement along the branches of a scaffold tree suggests that the bioactivity encoded in the derived substructures is partially retained, e.g., potency may be lowered, and proceeds with the retention of biological relevance. This principle is analogous to fragmentbased drug discovery, ${ }^{10}$ and is based on the assumption that smaller scaffolds may transfer their biologically relevant characteristics to the resulting molecules into which they are incorporated. As such, scaffold trees may be alternatively constructed on the basis of bioactivity instead of chemical structure. ${ }^{11}$ These biology-guided scaffold trees offer different perspectives of chemical space by using bioactivi- 


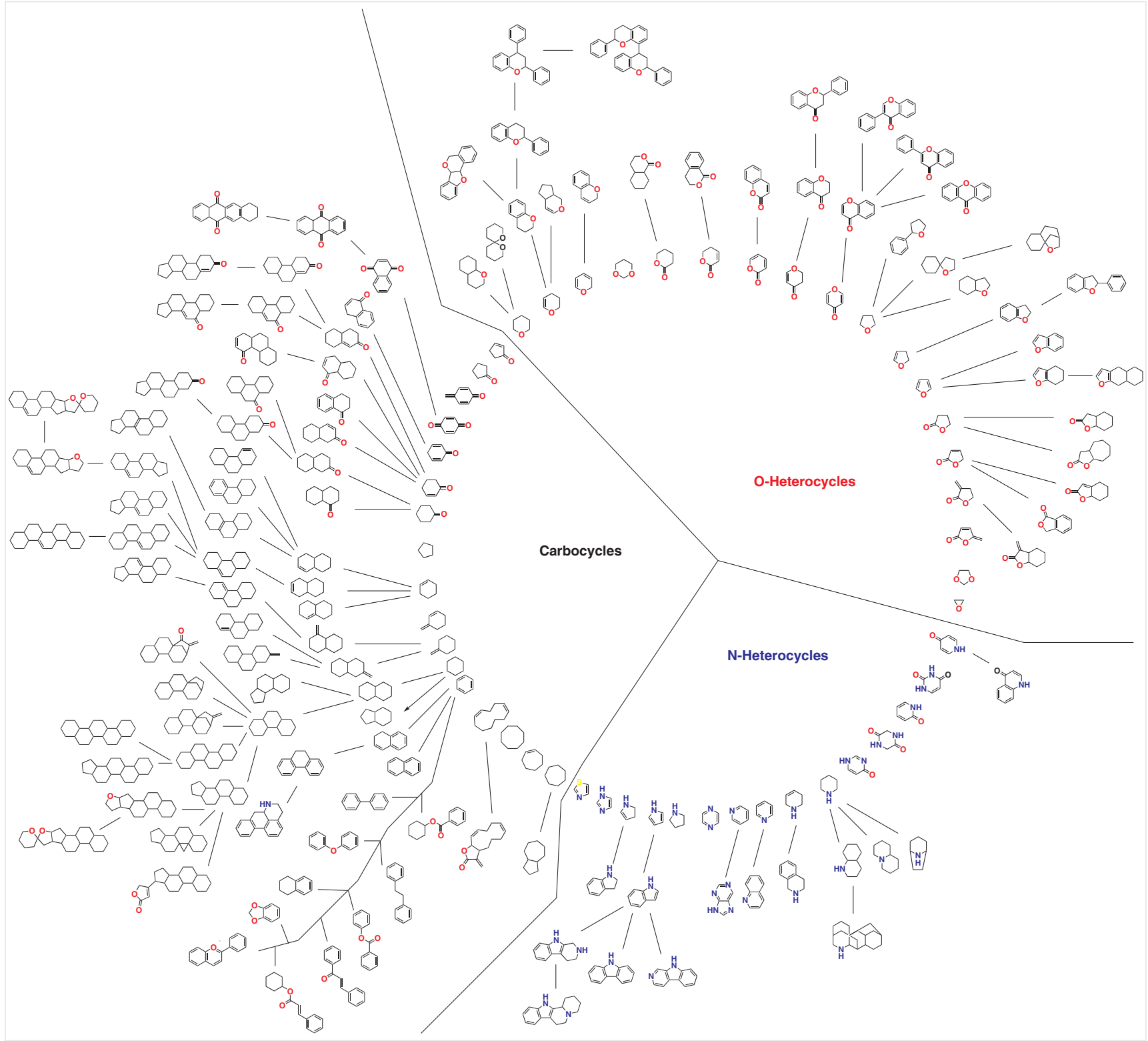

Figure 1 The classification of NP core scaffolds into a 'scaffold tree' as calculated by the SCONP algorithm

ty as the branch-constructing principle, and can lead to molecular scaffolds in which a particular kind of bioactivity is retained.

\section{Implications and Opportunities for Biolo- gy-Oriented Synthesis}

BIOS can be applied through SCONP to identify unknown, biologically relevant regions of chemical space. As bioactivity is guiding the selection of scaffolds, the resulting compound collections may be enriched in bioactivity, which in turn suggests that the size of the compound col- lections to be synthesized can be reduced. This principle compensates for the fact that the preparation of these compounds may be more elaborate compared to combinatorial libraries. Additionally, NP-inspired compounds may be less structurally complex when compared to the guiding NPs, resulting in reliable and efficient synthetic routes and retained bioactivity. Ultimately, BIOS aims to provide compounds which can be used to develop optimized structures based on a biologically relevant scaffold. This optimization can be achieved through established approaches and strategies of medicinal chemistry and may improve the potency or physicochemical properties of selected candidates depending on their intended use as lead for drug development 
or tool compounds for chemical biology. ${ }^{12}$ Thus, BIOS relies on the continuous development of novel and robust chemical methods that can be subsequently applied in the parallel preparation of compound collections with structurally complex scaffolds in a modular manner.

Preparing structurally simplified scaffolds, by following the BIOS process, may lead to the resulting molecules engaging multiple protein targets with similarly shaped ligand-binding sites. This potential promiscuity can be regarded as an advantage, as biological networks are often redundantly interconnected, suggesting that several modulating interactions are necessary for the desired biological effect. NPs have been selected and evolved for their ability to interact in a dynamic manner with such systems and these properties can be passed down to NP-inspired compounds through their chemical structures. Any selectivity issue which may arise can be addressed through established medicinal chemistry approaches, suggesting that BIOS offers unique opportunities for the preparation of molecules which may display poly-bioactivity profiles, and as such can be proven ideal tools for systems biology research (Scheme 1). To take full advantage of these poly-bioactive compounds, target-agnostic and cell-based phenotypic screenings can be undertaken; it is essential that these are coupled with effective strategies for target deconvolution, identification, and validation. ${ }^{8}$ Finally, the BIOS principles can be applied to non-natural-derived bioactive scaffolds and compound classes, as the approach is guided by the biological relevance itself and not its occurrence in nature.

\section{Applications of Biology-Oriented Synthesis}

The principles of BIOS can be applied to guide the design and preparation of NP-inspired molecular scaffolds employing different aspects of NPs as the guiding principle. For example, following the chemical structure of the core scaffold as the guiding principle may result in a distinct biologically relevant compound class compared to when utilizing bioactivity as a guiding principle. Employing these aspects in a complementary manner can result in the efficient preparation of biologically enhanced compound libraries and can lead to the discovery of structurally unprecedented bioactive small molecules.

\subsection{Chemical Structure and Bioactivity Guided Approaches}

Polycyclic scaffolds with a high degree of three-dimensionality can be prepared by the utilization of chemical cascade reactions. These scaffolds are useful synthetic tools to investigate the hypotheses generated by the application of the SCONP algorithm and the scaffold tree. Due to their<smiles>C=C(C)CCC[C@]1(C)CCC[C@H]2C1=CC[C@@H](C)[C@]2(C)C[C@H](O)C1=CC(=O)O[C@@H]1O</smiles><smiles>CC[C@H]1CN2CC[C@]3(C(=O)Nc4ccccc43)C2=C[C@@H]1/C(=C\OC)COC</smiles><smiles>O=C(O)[C@H]1[C@H]2C[C@@H]3c4[nH]c5ccccc5c4CCN3C[C@H]2CC[C@H]1O</smiles>

Scaffold tree lassification<smiles>C1CCC2CCCCC2C1</smiles>

Structurally simplified NP-inspired scaffolds

\section{BIOS}

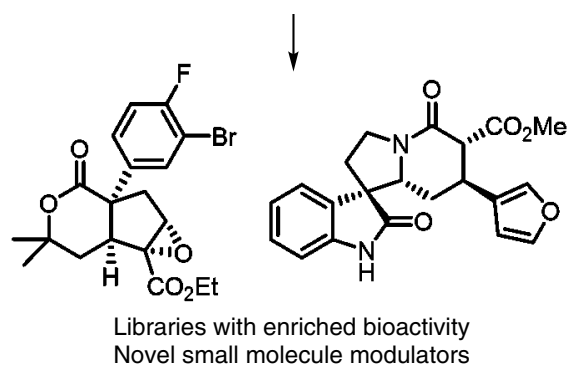

Scheme 1 BIOS overview. NPs are used to select simplified scaffolds that guide the preparation of compound libraries with enriched bioactivities, leading to the identification of novel small molecule modulators, which may be used as tools in chemical biology research.

modularity, cascade reactions facilitate the preparation of compound collections that are large enough for the application of BIOS, as the resulting scaffolds are already biologically relevant. Cascade reactions producing polycyclic scaffolds or fusing fragments require the use of structurally simple starting materials, in reduced quantities as well as in fewer handling and purification steps. As an example, a twelve-step reaction cascade sequence, including nine discrete chemical transformations, ${ }^{13}$ was used in the preparation of the structurally complex tetracyclic tetrahydroindoloquinolizines (Scheme 2, A). This scaffold resembles a 
<smiles>[10BH]c1ccc2[nH]cc(CCN)c2c1</smiles>

B<smiles>CC(=O)C=Cc1coc2ccccc2c1=O</smiles><smiles>C1=NCCc2ccccc21</smiles>

<smiles>[R]c1cc(C(=O)C2CC(C(=O)O)[C@@]3(CCc4c3[nH]c3ccc([125I])cc43)C2)c(O)c([2H])c1[2H]</smiles>

via 12-step reaction cascade



structurally simplified scaffold four-fold potency increase

Scheme 2 Cascade reactions are ideal for the application of BIOS. A) A structurally complex polycyclic scaffold was prepared by using a twelve-step reaction cascade. B) An enantioselective reaction was developed to prepare structurally simplified analogues resulting in the identification of mitosis modulators with up to a four-fold increase in potency.

number of polycyclic indole alkaloid structures. ${ }^{14}$ This cascade was further utilized to prepare a focused compound collection, which was screened for mitosis modulation, as previously reported indole-alkaloid-based molecules were reported to affect this process. ${ }^{15,16}$ Arrested mitosis and multipolar mitotic spindles were observed using confocal microscopy imaging of recombinant cells, revealing at least two compounds with low micromolar effective concentrations. The centrosomal protein NPM1, which is a centrosome duplication regulator, ${ }^{17}$ and its associated nuclear export factor $\mathrm{Crm} 1^{18}$ were identified as the biological targets through a chemical proteomics investigation using activitybased pull-down probes followed by MS determination or immunoblotting. Further evidence of target engagement was demonstrated by using knockdown cell lines. These novel inhibitors were used to guide the design of a focused structurally simplified collection, prepared by a newly developed enantioselective synthetic route (Scheme 2, B). ${ }^{19}$ Subsequent screening for mitosis modulation led to the identification of an analogue with a fourfold increased potency. This finding provided further evidence that BIOS can be applied in non-naturally occurring yet biologically relevant molecules as well.

Alternative, yet complementary to linear cascades, branching reaction cascades are highly efficient synthesis tools, which can be used for the preparation of diverse molecular scaffolds using only a few common precursors. ${ }^{20}$ Intra- or intermolecular reactions can be used, involving a number of complexity generating branching cascades resulting in the formation of diverse and complex molecular architectures in a rapid and concise manner. ${ }^{21}$ Thus, branching cascades are fitting tools for the preparation of compound collections guided by BIOS. For example, a range of bi-nucleophilic reagents were used with a common starting material bearing multiple electrophilic positions.
Their reaction resulted in the formation of twelve discrete and structurally diverse unprecedented molecular scaffolds (Scheme 3), some of which were NP-related. ${ }^{22}$

Iridoids are a class of structurally complex NPs, which have been reported to exhibit neuroprotective or neuritegrowth-promoting properties. ${ }^{23,24}$ The BIOS approach was applied to the iridoid scaffold in order to develop small molecules aimed at the study and treatment of neurodegenerative diseases..$^{25-27}$ A sequence involving a [3+2] cycloaddition and a regioselective Baeyer-Villiger oxidation gave

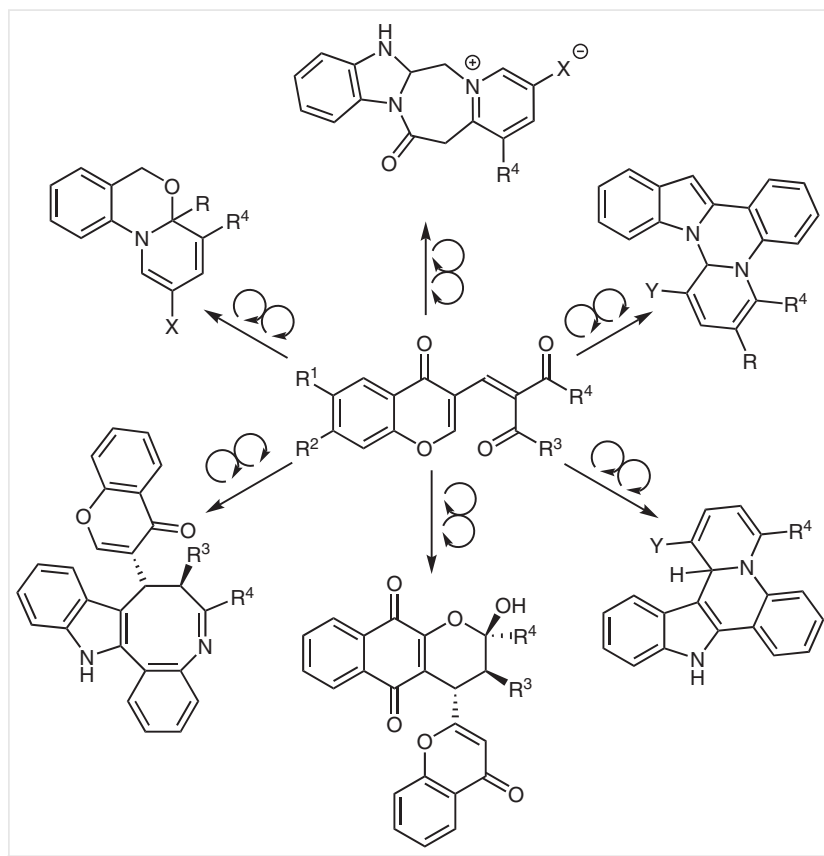

Scheme 3 Branching cascades are equally useful synthetic tools for the preparation of diverse and structurally complex molecules. 
access to two molecular scaffolds of the general iridoid class (Scheme 4). ${ }^{28}$ Subsequent development of an enantioselective route, followed by individual scaffold derivatization steps, led to the preparation of a collection of 54 molecules This collection was screened for activity by using a primary hippocampal neuron outgrowth assay and a mouse embryonic stem cell motor neuron growth assay, leading to the identification of novel active compounds with neuritegrowth-promoting properties. These compounds displayed different patterns of neurite growth promotion and may be used as biological tools to investigate these neurodevelopmental processes further.
A

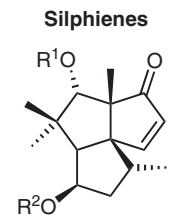

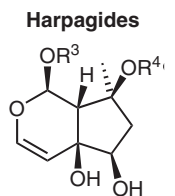

B

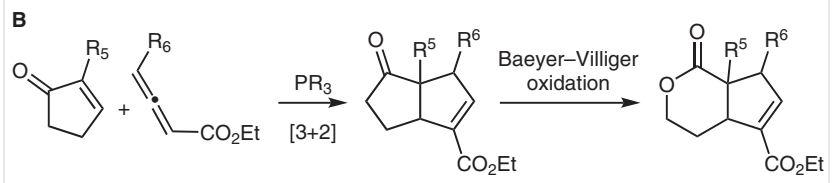

C

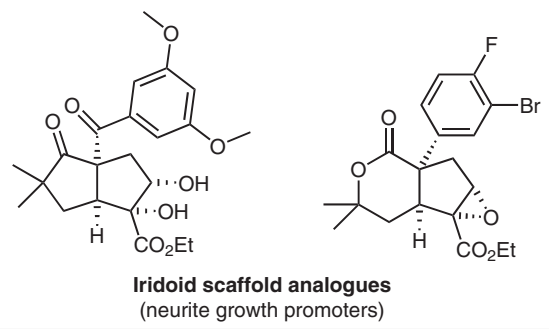

Scheme 4 Irridoid-inspired design and preparation of a neuritegrowth-promoting compound class: A. examples of iridoid NPs; B. chemical synthesis of iridoid scaffold analogues and related molecules; C. compounds that display neurite growth properties.

Similarly, secoyohimbane NPs (Scheme 5) have also been reported to possess neurite-growth-promoting properties. ${ }^{29-31}$ Their core scaffold is characterized by a high degree of three-dimensionality and consists of four connected rings and a spiral center. The development of an organocatalytic, enantioselective synthetic route towards secoyohimbane analogues gave access to a collection of 56 compounds. Although structurally simplified compared to the guiding NPs, five of these compounds exhibited neuritegrowth-promoting properties when using either the hippocampal neurite outgrowth or the mouse embryonic stem cell motor neurite growth assays. ${ }^{32}$ These findings provided further validation for BIOS as a chemocentric hypothesisgenerating approach, which can lead to the identification of bioactive small molecules.
A<smiles>CC[C@H]1CN2CC[C@]3(C(=O)Nc4ccccc43)[C@@H]2C[C@@H]1/C(=C\OC)C(=O)O</smiles>

B<smiles>[R]C=CC=CC(=O)OC(c1ccccc1)(c1ccccc1)C1CCCN1</smiles>

C<smiles>COC(=O)[C@@H]1C(=O)N2CC[C@@]3(C(=O)Nc4ccccc43)[C@H]2C[C@@H]1c1ccoc1</smiles><smiles>CC(=O)[C@H]1C(=O)N2CC[C@@]3(C(=O)Nc4ccccc43)C2C[C@H]1c1ccc2ccccc2c1</smiles>

Secoyohimbane scaffold analogues acting as neurite growth promoters

Scheme 5 Neurite-growth-promoting compounds inspired by the secoyohimbane scaffold: A) rychnophylline: a secoyohimbane alkaloid; B) accessing the secoyohimbane scaffold through an organocatalytic, enantioselective synthesis; C) secoyohimbane analogues with neurite growth properties.

\subsection{Natural-Product-Derived Fragment-Based Approaches}

Fragments are molecules with low molecular weight ${ }^{33}$ that have been shown to expedite the exploration of chemical space. ${ }^{34}$ Earlier attempts at fragment-based drug discovery ${ }^{10}$ utilized compound libraries where the fragments are derived from known drugs, covering already known portions of chemical space ${ }^{35,36}$ and including a low number of molecular structures with a high degree of three-dimensionality. ${ }^{37}$

Natural products are characterized by a high degree of three-dimensional character. Furthermore, they occupy biologically relevant portions of chemical space that are often not covered by synthetically accessible compounds, ${ }^{38}$ as NPs are biologically prevalidated by nature. Additionally, NP-derived fragments may display different biologically relevant profiles compared to synthetically accessible fragments, as suggested by the chemoinformatic analyses of natural product structures based on fragment likeness. ${ }^{39,40}$ Suitable NP-derived fragments can be selected by the SCONP algorithm using additional filtering algorithms. A virtual set of NP-derived fragments was shown to have a high degree of three-dimensional character, structural complexity, and diversity, and occupied different portions of chemical space compared to a set of commercially available fragments. ${ }^{41}$ Additionally, their distribution of physical properties was found to be similar to that of the guiding 

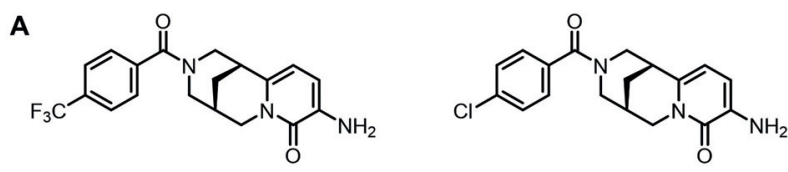

B

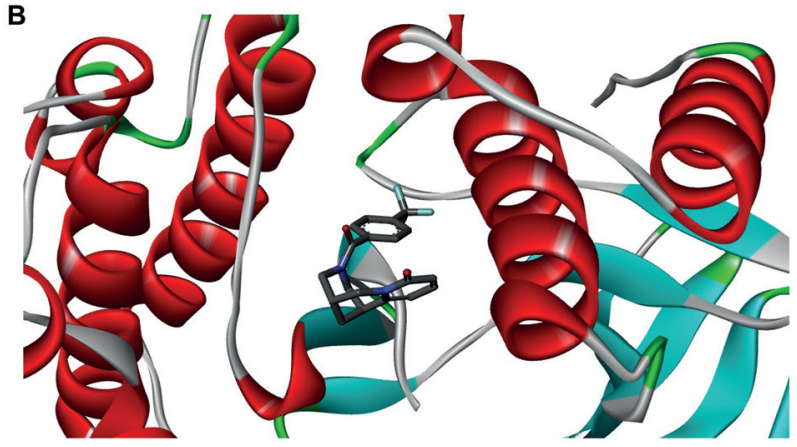

Figure 2 Natural-product-derived fragments identified as novel inhibitors of the p38a MAP kinase: A) examples of two different fragment classes; B) crystal structure of the p38a MAP kinase with a fragment bound at the allosteric site of the protein (PDB: 4EH9).

NPs. Thus, NP-derived fragments can be used to identify compounds with unprecedented chemotypes, which act as modulators of known biological targets. For example, derivatives of the structurally complex, bicyclic cytisinesparteine scaffold were found to be allosteric inhibitors of the p38a MAP kinase (Figure 2). ${ }^{41}$ These findings suggested that the BIOS principles can be expanded to include NPderived fragments as simplified substructures of NPs, with applications in fragment-based ligand discovery. These fragments are synthetically more tractable when compared to the guiding NPs, which may require multistep procedures for their preparation. Thus, compound libraries comprised of NP-derived fragments can be used to overcome limitations associated with synthetic tractability of certain NPs and efficient methodologies for the preparation of such fragments are in high demand.

Molecular scaffolds which include NP-derived fragments may result in compound collections with limited structural diversity, due to their predefined structures. To overcome this potential limitation, a common starting material can be used that combines an embedded NP fragment with orthogonally reactive functional groups. These groups can undergo different chemical transformations when treated with different catalysts or reagents and afford structurally diverse compounds which include NP-derived fragments in their molecular scaffolds. Versatile chemical methodologies which have the potential to proceed via multiple divergent reaction pathways are needed for the successful application of this approach. These reagentdirected syntheses can give access to compound collections with enhanced structural complexity and diversity as well as additional biological relevance instilled by the incorporation of the NP-derived fragment.
The oxindole fragment forms part of a number of diverse NPs exhibiting different bioactivities ${ }^{42,43}$ and is also incorporated in a number of compounds included in medicinal chemistry and drug discovery projects. ${ }^{44,45}$ Harnessing the versatility of gold-catalyzed reactions, a common oxindole-derived 1,6-enyne starting material was used to give rise to three distinct molecular scaffolds with NP features (Scheme 6, A). The regio-reactivity of the enyne functionality was modulated by the source of gold and the properties of the ligand used in each case, ${ }^{42,46,47}$ allowing the orthogonal preparation of each scaffold on demand leading to the divergent formation of a skeletally diverse collection of 60 compounds. In order to investigate the biological relevance and potential activity of these compounds, cellular assays monitoring biological pathways, rather than individual targets, were employed.

The Hedgehog (Hh) pathway is a conserved signaling pathway involved in a number of processes such as cell proliferation and differentiation and tissue regeneration and repair, and has been associated with conditions such as birth defects and cancer. ${ }^{48}$ Equally important, the Wnt signaling pathway is implicated in processes such as cell proliferation and migration, tissue regeneration, cell polarity, as well as stem cell renewal, playing a key role in the proliferation of malignant tissue. ${ }^{49}$ Autophagy is a catabolic process playing an integral part in cell homeostasis. This process involves the self-degradation of cellular components such as damaged organelles and denatured proteins, and is thought to act as a survival mechanism for cancer cells undergoing metabolic stress caused by a lack of nutrients or oxygen and increased energy demand. Inhibition of the autophagy process has recently emerged as a potential strategy for targeting cancer. ${ }^{50}$ By utilizing cellular assays that monitor these processes, structurally unprecedented and selective inhibitors of the Hedgehog and Wnt signaling pathways, the autophagy process, as well as cellular proliferation were revealed. Two compounds displayed low micromolar inhibitory activity of the Hh pathway, yet did not interfere with cell viability (Scheme 6, B). Additional biological characterization demonstrated that these compounds act as reversible binders of the transmembrane protein Smoothened (Smo), which is an activator of the $\mathrm{Hh}$ pathway. A different oxindole-derived compound was shown to have low micromolar inhibitory activity against the Wnt pathway. This selective and structurally novel inhibitor did not interfere with the Hh pathway (Scheme 6, B). A structurally distinct compound was revealed to inhibit the autophagy process selectively. Additionally, a novel cancer cell proliferation modulator was also identified by using an imaging assay which monitors cell confluency. This structurally distinct compound did not exhibit any activity in the Hh and Wnt or autophagy assays.

Following a different synthetic approach and working towards the application of these principles, an asymmetric 1,3-dipolar cycloaddition of azomethine ylides with various 


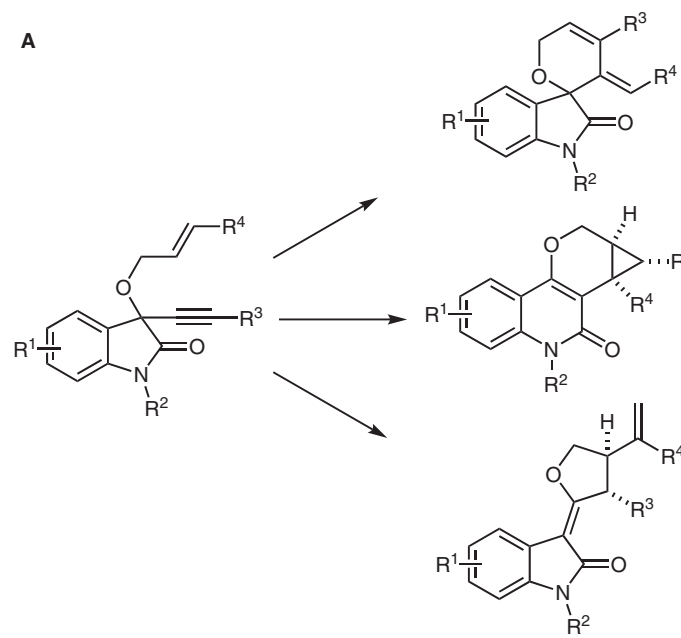

B<smiles>COc1ccc(Cn2c(=O)c3c(c4ccccc42)OC[C@H]2[C@H](C)[C@]32c2ccccc2)cc1</smiles><smiles>C[C@H]1[C@H]2COc3c(c(=O)n(C)c4ccc(Cl)cc34)[C@]12c1ccccc1</smiles>

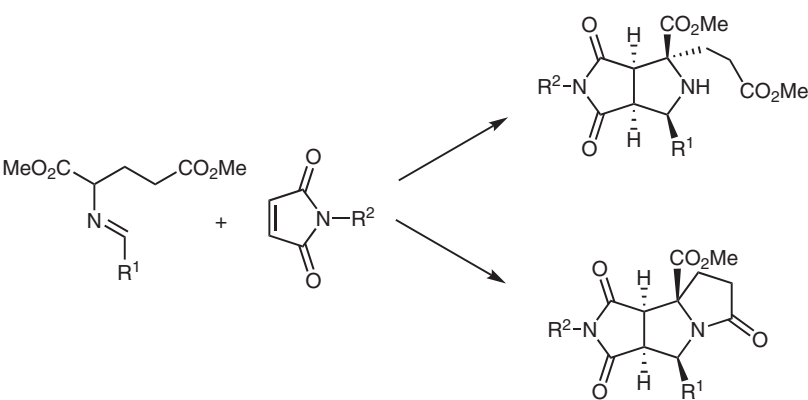

Wnt pathway inhibitor $\mathrm{IC}_{50}=4.2 \mu \mathrm{M}$

$$
\text { autophagy inhibitor }
$$
$\mathrm{IC}_{50}=4.8 \mu \mathrm{M}$<smiles></smiles><smiles>C=C(C)[C@@H]1CO/C(=C2/C(=O)N(Cc3ccccc3)c3ccccc32)[C@H]1C</smiles>

cell proliferation inhibitor ${ }^{\prime} C_{50}=15.4 \mu \mathrm{M}$

Hh pathway inhibitor IC $_{50}=2.75 \mu \mathrm{M}$

Novel small molecules with divergent biological activities

Scheme 6 NP-derived fragments may enhance biological relevance when embedded in molecular scaffolds. A) Scaffold-divergent synthesis led to the identification of skeletally different compounds from a common starting material, with diverse bioactivities. B) The enantioselective preparation of scaffolds containing pyrrolizidine fragments resulted in the identification of compounds with antibacterial properties.

olefins was employed to prepare a compound collection with a bicyclic scaffold containing the pyrrolizidine fragment. ${ }^{51}$ This NP-derived fragment is encountered in compounds with bioactivities ranging from insecticides to carcinogens and other alkaloid NPs. ${ }^{52,53}$ Employing glutamatederived imines and maleimide derivatives in the presence of a chiral copper-ferrocene catalyst complex, followed by an intramolecular lactamization, this new synthetic methodology gave access to a collection of 30 compounds. Selected compounds of this collection exhibited antibacterial activity, inhibiting the growth of the pathogen P. falciparum whilst others were revealed to act as Hedgehog pathway inhibitors with activities in the low micromolar range (Scheme 6, B). These findings demonstrate that synthetic approaches which incorporate NP-derived fragments can be applied to prepare biologically relevant compound collections. These distinct and structurally diverse small molecules may act as selective modulators of different biological processes and signaling pathways, with direct applications in chemical biology research.

The preparation of compound libraries with molecular scaffolds that have only certain common fragments, yet do not share the whole core scaffold with the guiding NPs, may still lead to the identification of biologically relevant small molecules. This observation suggests that novel molecular scaffolds may inherit the physiochemical and bioactive properties of NPs, if they incorporate combinations of NPderived fragments. These combinations may give rise to novel chemical matter that is biologically relevant, but not linked to specific biological targets. Thus, the resulting 
chemotypes can be used to explore portions of chemical space which cannot be reached by NPs, since such fragment combinations may not be obtainable through biosynthetic pathways. Similar to the biological evaluation of newly discovered NPs, these compounds require more elaborate screening efforts and their potential would be best explored by the use of target agnostic cellular assays which follow phenotypic changes rather than individual biological targets.

These novel NP-derived fragment combinations require the development of enantioselective synthetic methods for the preparation of enantiopure compounds at will, since bioactivity is often correlated with the absolute configuration of specific NPs. For example, a hybrid scaffold with eight stereocenters, combining the tropane and pyrrolidine fragments, was accessed through a catalytic enantioselective cycloaddition in an enantiodivergent fashion. ${ }^{54}$ The tropane fragment has been the focus of medicinal chemistry studies and biological research, ${ }^{55,56}$ since it is abundantly encountered in a plethora of alkaloid NPs that exhibit a range of diverse biological effects. ${ }^{57-59}$ Similarly, the pyrrolidine fragment can be found in a variety of biologically relevant NPs - alkaloids and others - and forms part of approved drugs as well as research compounds. ${ }^{60} \mathrm{~A}$ chiral copper(I)-phosphine catalyst was used to kinetically resolve the racemic tropane starting material, which reacts with an azomethine ylide to form this pyrrotropane scaffold. The unreacted enantiomer of the tropane starting material can then be converted into a distinct analogue of the pyrrotro- pane scaffold in the presence of a different azomethine ylide and a racemic copper(I)-phosphine in a one-pot manner. The two analogues can then be purified via chromatography with only one purification step resulting in the isolation of two distinct compounds. Furthermore, reversing the addition of the two azomethine ylides leads to the formation of the opposite enantiomers, which can also be obtained on demand (Scheme 7, A). In a different example, the combination of the tropane and oxindole fragments was achieved in an enantioselective fashion by utilizing a bimetallic relay catalysis approach. ${ }^{61}$ Oximino-derived cyclic azomethine ylides reacted with oxindole-bearing olefins leading to the formation of spiro-tropoxindoles (Scheme 7, B). This specific fragment combination is precedented in NPs, which exhibit diverse bioactivities such as antiplasmodials and vasorelaxants. ${ }^{58,62}$ However, a catalytic enantioselective method for the preparation of this biologically relevant and structurally complex molecular scaffold had not been reported previously. Such methods provide the opportunity to explore adjacent portions of chemical space to those covered by the guiding NPs, through the preparation of analogues which cannot be obtained through biosynthesis alone.

By extension, non-natural yet NP-inspired scaffolds may also be prepared by minimal chemical modifications of the guiding NPs. For example, the use of regioselective ring contractions or expansions or intramolecular transformation of functional groups can give access to new molecular scaffolds from NPs in a direct manner. These compounds

A


stereodiverse pyrrolidine-tropanes

B<smiles></smiles><smiles>N#CC(=O)CC/C([Tl])=N\O</smiles>
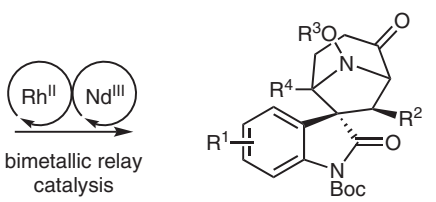

spiro-tropoxindoles

Scheme 7 A) One-pot enantiodivergent synthesis of hybrid NP-derived fragment scaffolds enabled by the kinetic resolution of tropanes with azomethine ylides catalyzed by a chiral copper(I)-phosphine complex. B) Enantioselective preparation of spiro-tropoxindoles, by means of bimetallic relay catalysis. 
may retain their biological relevance and properties ${ }^{63,64}$ and have the potential to fill in gaps in chemical space that have remained unexplored by nature. Due to their existing structural diversity, NPs can be used as the starting materials for such chemocentric approaches and they can prove to be ideal substrates for regio- and stereoselective transformations. ${ }^{65}$ The application of these principles was demonstrated by Hergenrother and co-workers, who utilized a ringdistortion strategy to prepare diverse, structurally complex, and distinct small molecules directly from guiding NPs in a rapid manner. ${ }^{66}$ This strategy allows the capitalization of NP properties, such as biologically relevant structural features, already bestowed by nature. ${ }^{65}$ In a similar manner, an oxazatwistane scaffold can be prepared directly from cinchona alkaloids via ring distortion. This class of NPs includes bioactive molecules such as quinine and quinidine (Figure 3). ${ }^{67}$ By utilizing late-stage $\mathrm{C}-\mathrm{H}$ functionalization, transformations to the resulting oxazatwistane scaffold, as well as metal-catalyzed coupling reactions allowed the preparation of a collection of about 50 analogues. These compounds were subjected to phenotypic and targetagnostic cellular assays leading to the identification of submicromolar inhibitors of starvation-induced autophagy (Figure 3). These compounds represent structurally unprecedented autophagy modulators ${ }^{68}$ and their bioactivity profile is distinct from that of the guiding NPs, as none of quinine, quinidine, or the unsubstituted oxazatwistane scaffold was found to exhibit this inhibitory activity at the tested concentration.
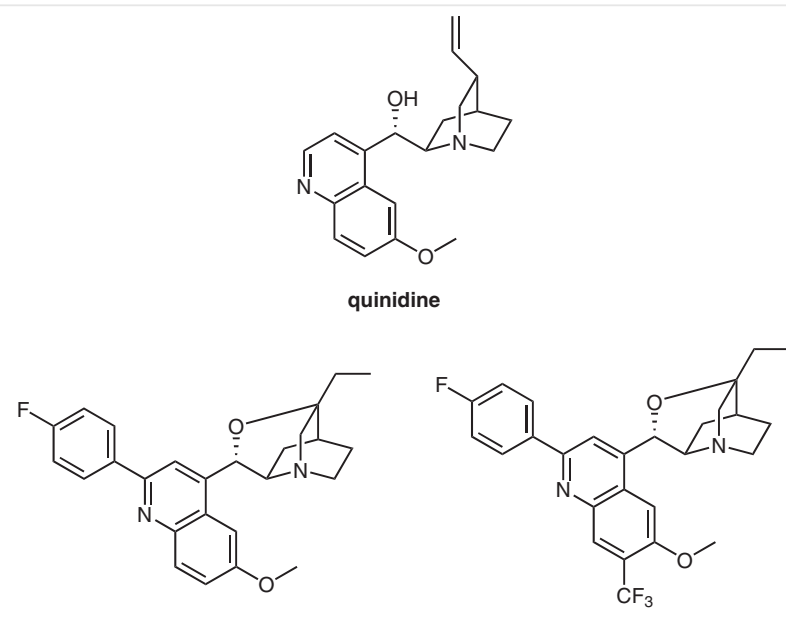

cinchona alkaloid derived oxazatwistane-autophagy inhibitors

Figure 3 Cinchona alkaloid natural products such as quinidine can be directly transformed into novel scaffolds such as the oxazatwistane. Late-stage functionalization enabled the preparation of substituted oxazatwistanes, which were identified as submicromolar inhibitors of autophagy.

\section{Conclusions and Outlook}

Small molecules are excellent tools for the study and elucidation of complex biological mechanisms, as their mode of action can be conditional, tunable, reversible, and rapid. Thus, design principles and methodologies facilitating the charting and qualitative analysis of chemical space in a systematic manner enable the identification of biologically relevant chemical matter and unprecedented bioactive chemotypes. The 'structural classification of natural products' (SCONP) algorithm enables the structural analyses of NP scaffolds, which represent prevalidated and biologically relevant spots in chemical space. As such, SCONP can be a hypothesis-forming tool, guiding the design and preparation of compound libraries which have been demonstrated to possess enriched bioactivity and give rise to potent and selective modulators for established biological targets. These compounds have also been used as tools for chemical biology research, leading to the identification of novel biological targets, or may be used as tools for the development of lead compounds for medicinal chemistry research. While BIOS collections may contain fewer members, the compounds prepared following BIOS design principles exhibit a higher degree of three-dimensional character compared to conventional combinatorial libraries. Furthermore, their concise syntheses enable the modular modification of functional groups, resulting in sufficient substituent diversity, which is crucial for the development of specific selectivity profiles of the corresponding small molecule modulators. Although additional synthetic effort is required for their preparation, BIOS collections display higher hit rates compared to combinatorial libraries, due to their enhanced biological relevance. Evidently, the development and application of novel synthetic methodologies granting access to NP-inspired molecular scaffolds in an efficient and reliable fashion lie at the core of BIOS, which is a chemocentric approach for the identification of biologically relevant chemical matter.

These principles were also applied to fragments that have been shown to expedite the exploration of chemical space and are significantly more synthetically tractable. Specifically NP-derived fragments were included in the SCONP algorithm, thus expanding the scope of BIOS and merging this approach with fragment-based compound discovery. This combination provides an additional tool for the identification of biologically relevant compounds. The full potential of these unprecedented molecules can be demonstrated by the use of unbiased screening methods, such as cell-based and target-agnostic phenotypic assays, as demonstrated by the identification of novel and selective modulators of the Hedgehog and Wnt signaling pathways, or the autophagy process.

Lastly, BIOS is an approach inspired by the evolutionary conservation of molecular architectures observed in nature, and relies on the biological relevance and prevalidation of 
NPs. BIOS libraries have been shown to have enhanced biological relevance, leading to the discovery of small molecule modulators with unprecedented chemotypes with high hit rates. Therefore, following the BIOS design principles enables overcoming limitations such as synthetic tractability in the study of NP-inspired compounds and provides new opportunities for organic synthesis aligned with biological research.

\section{References}

(1) Zamir, E.; Bastiaens, P. I. H. Nat. Chem. Biol. 2008, 4, 643.

(2) Dobson, C. M. Nature 2004, 432, 824.

(3) Gao, M.; Skolnick, J. PLoS Comput. Biol. 2013, 9, e1003302.

(4) Patel, S. C.; Bradley, L. H.; Jinadasa, S. P.; Hecht, M. H. Protein Sci. 2009, 18, 1388.

(5) Sadreyev, R. I.; Grishin, N. V. BMC Struct. Biol. 2006, 6, 6.

(6) Newman, D. J.; Cragg, G. M. J. Nat. Prod. 2007, 70, 461.

(7) Lovering, F.; Bikker, J.; Humblet, C. J. Med. Chem. 2009, 52, 6752.

(8) Ziegler, S.; Pries, V.; Hedberg, C.; Waldmann, H. Angew. Chem. Int. Ed. 2013, 52, 2744.

(9) Koch, M. A.; Schuffenhauer, A.; Scheck, M.; Wetzel, S.; Casaulta, M.; Odermatt, A.; Ertl, P.; Waldmann, H. Proc. Natl. Acad. Sci. U.S.A. 2005, 102, 17272.

(10) Murray, C. W.; Rees, D. C. Nat. Chem. 2009, 1, 187.

(11) Renner, S.; van Otterlo, W. A. L.; Dominguez Seoane, M.; Mocklinghoff, S.; Hofmann, B.; Wetzel, S.; Schuffenhauer, A.; Ertl, P.; Oprea, T. I.; Steinhilber, D.; Brunsveld, L.; Rauh, D.; Waldmann, H. Nat. Chem. Biol. 2009, 5, 585.

(12) Frye, S. V. Nat. Chem. Biol. 2010, 6, 159.

(13) Dückert, H.; Pries, V.; Khedkar, V.; Menninger, S.; Bruss, H.; Bird, A. W.; Maliga, Z.; Brockmeyer, A.; Janning, P.; Hyman, A.; Grimme, S.; Schürmann, M.; Preut, H.; Hübel, K.; Ziegler, S.; Kumar, K.; Waldmann, H. Nat. Chem. Biol. 2012, 8, 179.

(14) Ishikura, M.; Yamada, K.; Abe, T. Nat. Prod. Rep. 2010, 27, 1630.

(15) Hung, D. T.; Jamison, T. F.; Schreiber, S. L. Chem. Biol. 1996, 3 , 623.

(16) Peterson, J. R.; Mitchison, T. J. Chem. Biol. 2002, 9, 1275.

(17) Lim, M. J.; Wang, X. W. Cancer Detect. Prev. 2006, 30, 481.

(18) Hutten, S.; Kehlenbach, R. H. Trends Cell Biol. 2007, 17, 193.

(19) Eschenbrenner-Lux, V.; Küchler, P.; Ziegler, S.; Kumar, K.; Waldmann, H. Angew. Chem. Int. Ed. 2014, 53, 2134.

(20) Enders, D.; Grondal, C.; Hüttl, M. R. M. Angew. Chem. Int. Ed. 2007, 46, 1570.

(21) Nielsen, T. E.; Schreiber, S. L. Angew. Chem. Int. Ed. 2008, 47, 48.

(22) Liu, W.; Khedkar, V.; Baskar, B.; Schürmann, M.; Kumar, K. Angew. Chem. Int. Ed. 2011, 50, 6900.

(23) Butler, M. S. Nat. Prod. Rep. 2008, 25, 475.

(24) Prisinzano, T. E. J. Nat. Prod. 2009, 72, 581.

(25) Jessen, H. J.; Schumacher, A.; Shaw, T.; Pfaltz, A.; Gademann, K. Angew. Chem. Int. Ed. 2011, 50, 4222.

(26) Kuai, L.; Wang, X.; Madison, J. M.; Schreiber, S. L.; Scolnick, E. M.; Haggarty, S. J. ACS Chem. Neurosci. 2010, 1, 325.

(27) Bauer, A. J.; Stockwell, B. R. Chem. Rev. 2008, 108, 1774.

(28) Dakas, P.-Y.; Parga, J. A.; Höing, S.; Schöler, H. R.; Sterneckert, J.; Kumar, K.; Waldmann, H. Angew. Chem. Int. Ed. 2013, 52, 9576.

(29) Praveen Kumar, V.; Gajendra Reddy, R.; Vo, D. D.; Chakravarty, S.; Chandrasekhar, S.; Grée, R. Bioorg. Med. Chem. Lett. 2012, 22, 1439.
(30) Cheng, X.; Harzdorf, N.; Khaing, Z.; Kang, D.; Camelio, A. M.; Shaw, T.; Schmidt, C. E.; Siegel, D. Org. Biomol. Chem. 2012, 10, 383.

(31) Rawat, M.; Gama, C. I.; Matson, J. B.; Hsieh-Wilson, L. C. J. Am. Chem. Soc. 2008, 130, 2959.

(32) Antonchick, A. P.; López-Tosco, S.; Parga, J.; Sievers, S.; Schürmann, M.; Preut, H.; Höing, S.; Schöler, H. R.; Sterneckert, J.; Rauh, D.; Waldmann, H. Chem. Biol. 2013, 20, 500.

(33) Chessari, G.; Woodhead, A. J. Drug Discov. Today 2009, 14, 668.

(34) Roughley, S. D.; Hubbard, R. E. J. Med. Chem. 2011, 54, 3989.

(35) Siegel, M. G.; Vieth, M. Drug Discov. Today 2007, 12, 71.

(36) Babaoglu, K.; Shoichet, B. K. Nat. Chem. Biol. 2006, 2, 720.

(37) Hung, A. W.; Ramek, A.; Wang, Y.; Kaya, T.; Wilson, J. A.; Clemons, P. A.; Young, D. W. Proc. Natl. Acad. Sci. U.S.A. 2011, $108,6799$.

(38) Grabowski, K.; Baringhaus, K.-H.; Schneider, G. Nat. Prod. Rep. 2008, 25, 892.

(39) Congreve, M.; Carr, R.; Murray, C.; Jhoti, H. Drug Discov. Today 2003, 8, 876.

(40) Köster, H.; Craan, T.; Brass, S.; Herhaus, C.; Zentgraf, M.; Neumann, L.; Heine, A.; Klebe, G. J. Med. Chem. 2011, 54, 7784.

(41) Over, B.; Wetzel, S.; Grütter, C.; Nakai, Y.; Renner, S.; Rauh, D.; Waldmann, H. Nat. Chem. 2013, 5, 21.

(42) Furstner, A. Chem. Soc. Rev. 2009, 38, 3208.

(43) Blunt, J. W.; Copp, B. R.; Munro, M. H. G.; Northcote, P. T.; Prinsep, M. R. Nat. Prod. Rep. 2005, 22, 15.

(44) Galliford, C. V.; Scheidt, K. A. Angew. Chem. Int. Ed. 2007, 46, 8748.

(45) Ding, K.; Lu, Y.; Nikolovska-Coleska, Z.; Wang, G.; Qiu, S.; Shangary, S.; Gao, W.; Qin, D.; Stuckey, J.; Krajewski, K.; Roller, P. P.; Wang, S. J. Med. Chem. 2006, 49, 3432.

(46) Pérez-Galán, P.; Martin, N. J. A.; Campaña, A. G.; Cárdenas, D. J.; Echavarren, A. M. Chem. Asian J. 2011, 6, 482.

(47) López-Carrillo, V.; Huguet, N.; Mosquera, Á.; Echavarren, A. M. Chem. Eur. J. 2011, 17, 10972.

(48) Briscoe, J.; Therond, P. P. Nat. Rev. Mol. Cell Biol. 2013, 14, 416.

(49) Anastas, J. N.; Moon, R. T. Nat. Rev. Cancer 2013, 13, 11.

(50) Choi, K. S. Exp. Mol. Med. 2012, 44, 109.

(51) Jia, Z. J.; Shan, G.; Daniliuc, C. G.; Antonchick, A. P.; Waldmann, H. Angew. Chem. Int. Ed. 2018, 1.

(52) Edgar, J. A.; Molyneux, R. J.; Colegate, S. M. Chem. Res. Toxicol. 2015, $28,4$.

(53) Robertson, J.; Stevens, K. Nat. Prod. Rep. 2014, 31, 1721.

(54) Xu, H.; Golz, C.; Strohmann, C.; Antonchick, A. P.; Waldmann, H. Angew. Chem. Int. Ed. 2016, 55, 7761; Angew. Chem. 2016, 128, 7892.

(55) Singh, S. Chem. Rev. 2000, 100, 925.

(56) Carroll, F. I.; Runyon, S. P.; Abraham, P.; Navarro, H.; Kuhar, M. J.; Pollard, G. T.; Howard, J. L. J. Med. Chem. 2004, 47, 6401.

(57) Grynkiewicz, G.; Gadzikowska, M. Pharmacol. Rep. 2008, 60, 439.

(58) Cheenpracha, S.; Ritthiwigrom, T.; Laphookhieo, S. J. Nat. Prod. 2013, 76, 723 .

(59) O'Hagan, D. Nat. Prod. Rep. 1997, 14, 637.

(60) O'Hagan, D. Nat. Prod. Rep. 2000, 17, 435.

(61) Jia, Z. J.; Takayama, H.; Futamura, Y.; Aono, H.; Bauer, J. O.; Strohmann, C.; Antonchick, A. P.; Osada, H.; Waldmann, H. J. Org. Chem. 2018, 83, 7033.

(62) Zaima, K.; Koga, I.; Iwasawa, N.; Hosoya, T.; Hirasawa, Y.; Kaneda, T.; Ismail, I. S.; Lajis, N. H.; Morita, H. J. Nat. Med. 2013, 67, 9.

(63) Rafferty, R. J.; Hicklin, R. W.; Maloof, K. A.; Hergenrother, P. J. Angew. Chem. Int. Ed. 2014, 53, 220. 
Synthesis

(64) Garcia-Castro, M.; Zimmermann, S.; Sankar, M. G.; Kumar, K. Angew. Chem. Int. Ed. 2016, 55, 7586.

(65) Morrison, K. C.; Hergenrother, P. J. Nat. Prod. Rep. 2014, 31, 6.

(66) Huigens, R. W.; Morrison, K. C.; Hicklin, R. W.; Flood, T. A.; Richter, M. F.; Hergenrother, P. J. Nat. Chem. 2013, 5, 195.
(67) Amirkia, V.; Heinrich, M. Phytochem. Lett. 2014, 10, xlviii.

(68) Laraia, L.; Ohsawa, K.; Konstantinidis, G.; Robke, L.; Wu, Y.-W.; Kumar, K.; Waldmann, H. Angew. Chem. Int. Ed. 2017, 56, 2145. 